\title{
T cell progenitor therapy-facilitated thymopoiesis depends upon thymic input and continued thymic microenvironment interaction
}

\author{
Michelle J. Smith, ${ }^{1,2}$ Dawn K. Reichenbach,, ${ }^{1,2}$ Sarah L. Parker, ${ }^{3}$ Megan J. Riddle, ${ }^{1}$ \\ Jason Mitchell, ${ }^{2,3}$ Kevin C. Osum, ${ }^{2,3}$ Mahmood Mohtashami, ${ }^{4}$ Heather E. Stefanski, ${ }^{1}$ Brian T. Fife, ${ }^{2,3}$ \\ Avinash Bhandoola, ${ }^{5}$ Kristin A. Hogquist, ${ }^{2}$ Georg A. Holländer, ${ }^{6,7}$ Juan Carlos Zúñiga-Pflücker, ${ }^{4}$ \\ Jakub Tolar, ${ }^{1}$ and Bruce R. Blazar ${ }^{1,2}$ \\ 'Division of Blood and Marrow Transplantation, Department of Pediatrics, Masonic Cancer Center, University of Minnesota, \\ Minneapolis, Minnesota, USA. ${ }^{2}$ Center for Immunology, Department of Medicine, and ${ }^{3}$ Department of Medicine, \\ University of Minnesota Medical School, Minneapolis, Minnesota, USA. ${ }^{4}$ Sunnybrook Research Institute and Department \\ of Immunology, University of Toronto, Toronto, Ontario, Canada. ${ }^{5}$ T-Cell Biology and Development Unit, Laboratory of \\ Genome Integrity, Center for Cancer Research, National Cancer Institute, Bethesda, Maryland, USA. ${ }^{6}$ Department of \\ Biomedicine, University of Basel, Basel, Switzerland. 'Department of Paediatrics and Weatherall Institute of Molecular \\ Medicine, University of Oxford, Oxford, United Kingdom.
}

Infusion of in vitro-derived T cell progenitor (proT) therapy with hematopoietic stem cell transplant aids the recovery of the thymus damaged by total body irradiation. To understand the interaction between proTs and the thymic microenvironment, WT mice were lethally irradiated and given T celldeficient (Rag1-/-) marrow with WT in vitro-generated proTs, limiting mature T cell development to infused proTs. ProTs within the host thymus led to a significant increase in thymic epithelial cells (TECs) by day 21 after transplant, increasing actively cycling TECs. Upon thymus egress (day 28), proT TEC effects were lost, suggesting that continued signaling from proTs is required to sustain TEC cycling and cellularity. Thymocytes increased significantly by day 21 , followed by a significant improvement in mature $\mathrm{T}$ cell numbers in the periphery by day 35 . This protective surge was temporary, receding by day 60 . Double-negative 2 (DN2) proTs selectively increased thymocyte number, while DN3 proTs preferentially increased TECs and T cells in the spleen that persisted at day 60. These findings highlight the importance of the interaction between proTs and TECs in the proliferation and survival of TECs and that the maturation stage of proTs has unique effects on thymopoiesis and peripheral T cell recovery.

Conflict of interest: The authors have declared that no conflict of interest exists.

Submitted: November 30, 2016 Accepted: April 13, 2017 Published: May 18, 2017

\section{Reference information:} JCI Insight. 2017;2(10):e92056. https://doi.org/10.1172/jci. insight. 92056 .

\section{Introduction}

Allogeneic hematopoietic stem cell transplantation (HSCT) is the current standard of care for many malignant and nonmalignant hematopoietic diseases (1-4). Prior to infusion of the donor graft, the patient must go through a conditioning regimen involving chemotherapy or chemoradiotherapy. This conditioning inflicts severe damage on the thymic microenvironment and results in delayed peripheral CD $4^{+}$and $\mathrm{CD} 8^{+}$ $\mathrm{T}$ cell reconstitution (5-9), leaving the patient vulnerable to opportunistic infections (10-12). The elements of the thymic microenvironment most sensitive to damage from chemoradiotherapy are the thymic epithelial cells (TECs), and their loss is responsible for prolonged impairment of thymopoiesis following transplant, which depends on successful recruitment of progenitors from the BM graft $(13,14)$.

TECs are indispensable to T cell development in vivo (15). They support homing of progenitors to the thymus $(16,17)$, provide critical survival signals $(18,19)$, direct trafficking of progenitors between the cortex and the medulla (18), and regulate positive and negative selection (20) and egress of single-positive T cells from the thymus (18). Several strategies have been investigated preclinically to preserve TECs during pretransplant conditioning regimens, such as prophylactic administration of keratinocyte growth factor (KGF) (21) and KGF in combination with leuprolide acetate (22) or transient inhibition of $\mathrm{p} 53$ prior to irradiation (23). However, these approaches have not yet led to a rapid and full restoration of peripheral $\mathrm{T}$ cell recovery early after transplant. 
Importantly, TECs also depend on interactions with developing T cells in order to develop, proliferate, and persist $(15,24-26)$. Conditioning eliminates developing thymocytes, and, in order to generate new $\mathrm{T}$ cells, there is a reliance on HSC-derived progenitors to home to and differentiate to $\mathrm{T}$ cell progenitors (proTs) within the thymus. Therefore, we utilized in vitro culture systems that use OP9-DLI cells to generate large numbers of proTs for thymic reconstitution and to speed thymic regeneration. The OP9-DL1 system uses a mouse BM stromal cell line (OP9) expressing Delta-like 1 (D111), a Notch1 receptor ligand, to direct and support the differentiation of $\mathrm{T}$ cells from hematopoietic (or embryonic) stem/progenitor cells $(27,28)$, of both mouse and human origin, that range in maturation from CD4-CD8- double-negative 1 (DN1) to DN4 cells (27-29). Other approaches, such as TSt-4 cells expressing Notch ligands D111 or D114 (30) or feeder-free systems based on immobilized D114 (31), can be used to achieve similar differentiations. In an allogeneic mouse model of HSCT, proTs differentiated and expanded using these methods when transferred together with HSCT, increased thymopoiesis following transplant (30-32), and conferred protection against tumor challenge (33), without the requirement for MHC matching between the proT donor and recipient. Given that a potential therapy using OP9-DL1-derived proTs is being developed for clinical use, we were interested in the specific effects and the stage of differentiation of proTs prior to adoptive transfer on TEC regeneration in a posttransplant setting. To isolate and characterize these effects, we employed a congenic mouse transplant model in which the in vitro-derived proTs or proTs enriched in DN2 or DN3 cells are adoptively transferred with T cell-depleted (or -deficient) BM into lethally irradiated recipient mice. In this model, we showed that the compartment of the thymus influenced by adoptive transfer of proTs was dependent upon the maturation stage of the input population and that a key factor in the continued benefits derived from this therapy was ongoing recruitment of new progenitors from the BM.

\section{Results}

ProTs home to the thymus and immigrate to and persist in the periphery. The initial and rate-limiting step in T-lymphopoietic and TEC recovery after conditioning and transplant requires homing of donor progenitor cells to the thymus (34). Thus, we sought to assess the homing and persistence of proTs in our model as well as compare their migration on both healthy (nonirradiated) and irradiated thymic stromal cells. ProTs were generated from donors expressing a luciferase transgene and transplanted with WT congenically marked BM cells into lethally irradiated recipients. The adoptively transferred cells were predominantly at the DN3 stage of development at the time of transplant (Figure 1A), and photoluminescent signal was monitored and quantified weekly (Figure 1, B and C). We observed a peak in both total body and thymically located signal at week 3 after transplant (Figure 1D).

The degree and nature of thymic recovery depends on the subset of DN T cells that are transplanted. Given that previous work on proT therapy indicated that the DN2 subset of proTs was most effective at increasing thymic size after allotransplant (32), we wanted to examine the differential thymus homing effects of the two dominant DN populations that are produced in OP9-DL1 cell cocultures with our congenic model, $\mathrm{DN} 2$ and DN3, as each is attainable in required numbers for adoptive transfer in vivo. Under normal physiological conditions, DN2- and DN3-developing thymocytes are located within the cortical and subcapsular regions of the thymus, while interacting with cortical TECs (cTECs) and undergoing further differentiation into $\mathrm{TCR}^{+} \mathrm{CD} 4^{+} \mathrm{CD}^{+}$double-positive cells that then undergo positive selection. As the thymocytes progress through their maturational program, they upregulate CC-chemokine receptor 7 and move to the medulla, wherein medullary TECs (mTECs) direct negative selection. If progenitors survive negative selection and achieve a mature single-positive state, then they upregulate the sphingosine-1-phosphate receptor 1 , which directs their egress from the thymus into the periphery (35).

We next tested whether in vitro-derived proTs would interact with the thymic microenvironment in the same way and specifically determined if different developmental stage subsets would have divergent effects on the thymic microenvironment. In order to answer this question, we sorted DN2 and DN3 subpopulations from OP9-DL1 cocultures and transplanted these with congenic T cell-depleted BM cells into lethally irradiated B6 recipients (Figure 2A). Mice that received total body irradiation experienced an $80 \%$ to $90 \%$ loss of mTECs and cTECs as compared with nontransplanted controls (Figure 2, B and C, and Supplemental Figure 1; supplemental material available online with this article; https:// doi.org/10.1172/jci.insight.92056DS1). On day 21 after transplant, when proT numbers peak within the thymus, mice given DN3 proTs showed a significant increase in the numbers of mTECs (Figure 2B). In contrast, cTEC numbers were unchanged after injection of either DN2 or DN3 (Figure 2C). 


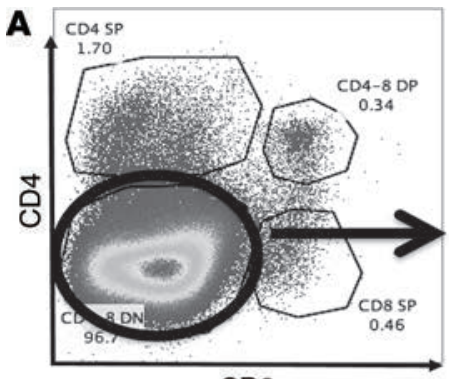

CD8

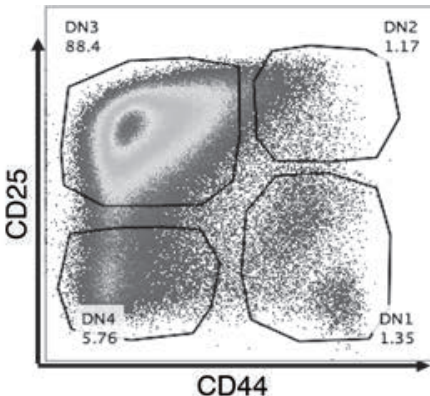

CD44

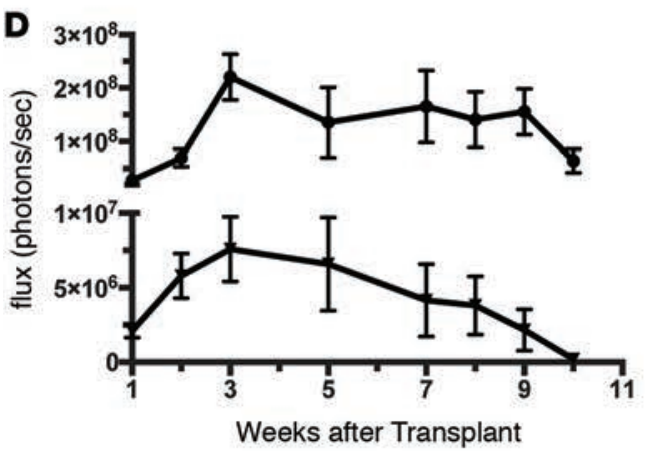

— Total Body

- Thymus

B
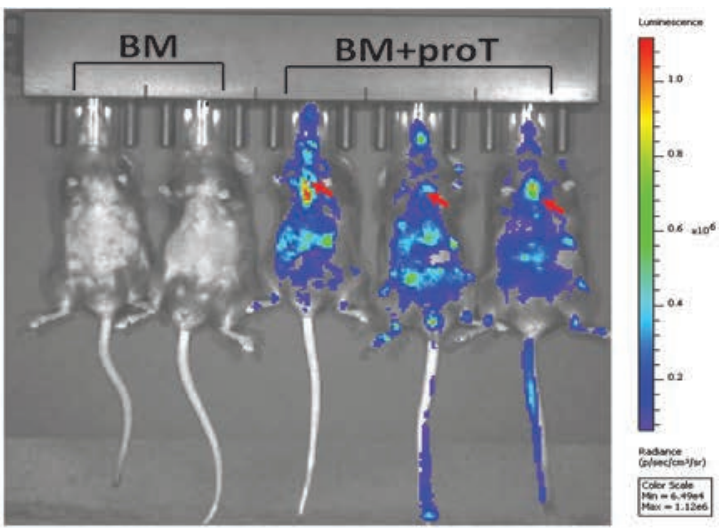

C
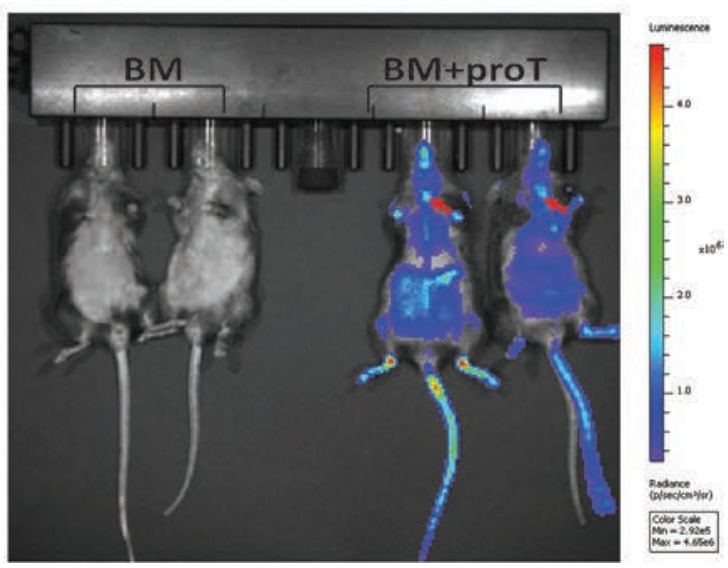

Figure 1. T cell progenitors home to the thymus but migrate with reduced velocity on irradiated stroma. Lethally irradiated B6 recipients were given congenically marked B6 BM with or without luciferase-positive progenitors (proTs). Animals were monitored using a Xenogen bioluminescent imager. Data are representative of 2 separate experiments, with 4-5 animals in each group. (A) Luciferase-positive proTs were predominantly at the double-negative 3 (DN3) stage at the time of transplant. Live cells were selected, and then DN3 cells were defined as (CD19, B220, Gr-1, CD11b, CD11c, Ter119, NK1.1)-CD45+CD4CD8 ${ }^{-C D 25}{ }^{+} C_{4} 44^{\prime}$. Luciferase signal visualized at (B) week 1 after transplant and (C) week 10 after transplant. Arrows indicate the location of the thymus.

(D) The total and thymically located luciferase signal measured weekly over 10 weeks after transplant.

Given the differential effect of these two subsets of DN cells on the thymic microenvironment and the known dependence of TECs on crosstalk with developing thymocytes (36-39), we sought to examine the location of the adoptively transferred DN2 and DN3 subsets within the thymus. On day 7 after proT engraftment, there were similar numbers of DN2 and DN3 proT-derived cells within the thymus, with the majority of them being located in the cortex as expected (Figure 2, D and E). On day 21, there were significantly more thymically located proTs in mice that received the DN2 cells compared with the BM-only group; the mice that received DN3 cells did not display a similar increase (Figure 3A), as measured by flow cytometry and immunofluorescence (Figure 2, D and E). The proTs in the group that received the DN3 subset were located primarily in the medulla, in contrast to proTs in the group that received the DN2 subset that remained primarily within the cortex (Figure 2, D and E).

Total thymic cellularity was increased on day 21 after transplant in the DN2 but not DN3 proT group (Figure 3A). Neither group of proT recipients had increased peripheral $\mathrm{T}$ cells in either the spleen or the lymph node $(\mathrm{LN})$ at this time point as compared with BM-only controls. The $\mathrm{T}$ cells that were present in the spleen at day 21 after transplant were overwhelmingly derived from the host, which is consistent with homeostatic expansion due to radiation-induced lymphopenia (Figure 3B). Given the dependence of TECs on interactions with developing thymocytes, the difference in localization between the DN2and DN3-derived proTs, which correlates with the increase in mTECs $\left(\mathrm{CD} 45^{-} \mathrm{EpCAM}{ }^{+} \mathrm{MHCII}{ }^{+} \mathrm{UEA} 1^{+}\right)$ in the group that received DN3 proTs, supports a hypothesis that adoptively transferred proTs improve long-term HSC-derived peripheral $\mathrm{T}$ cell reconstitution by improving thymopoiesis through interactions with mTECs. Similar to day 21, at day 60 after BM transplant (BMT), this effect was seen in the spleen (Figure 3D) but not in the peripheral LN (data not shown), indicating a defect in homing to the LN, 
A

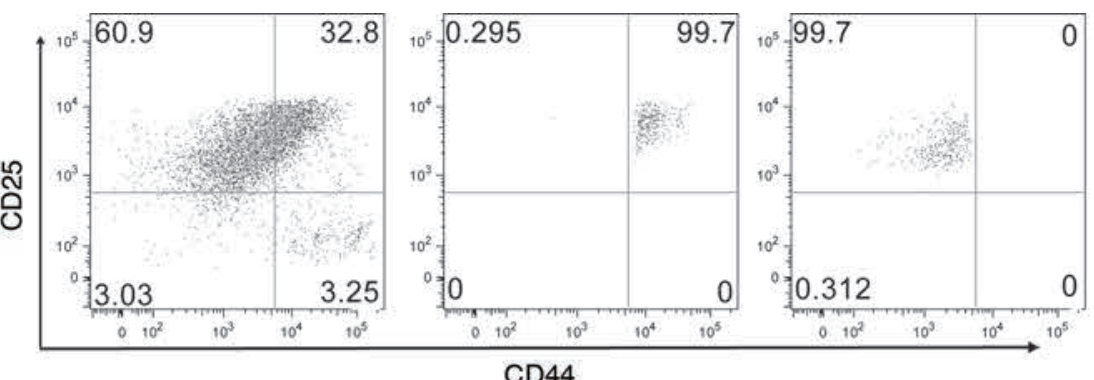

B

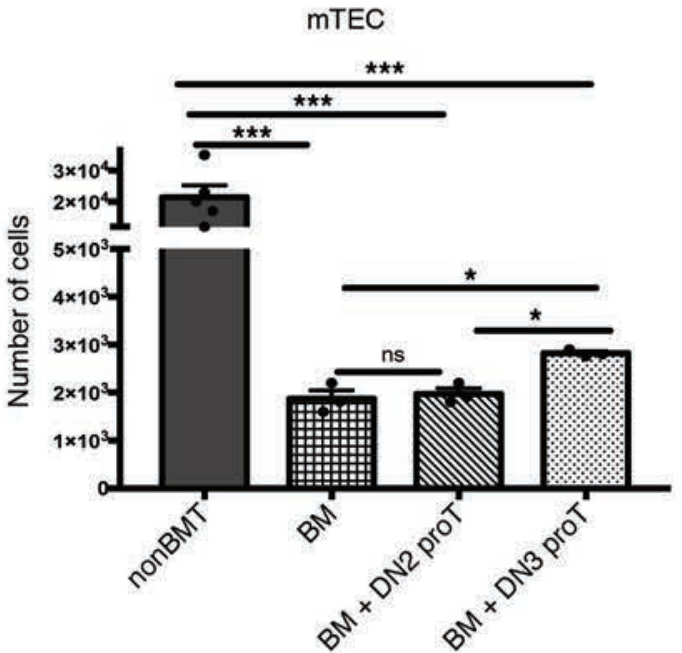

Day 7
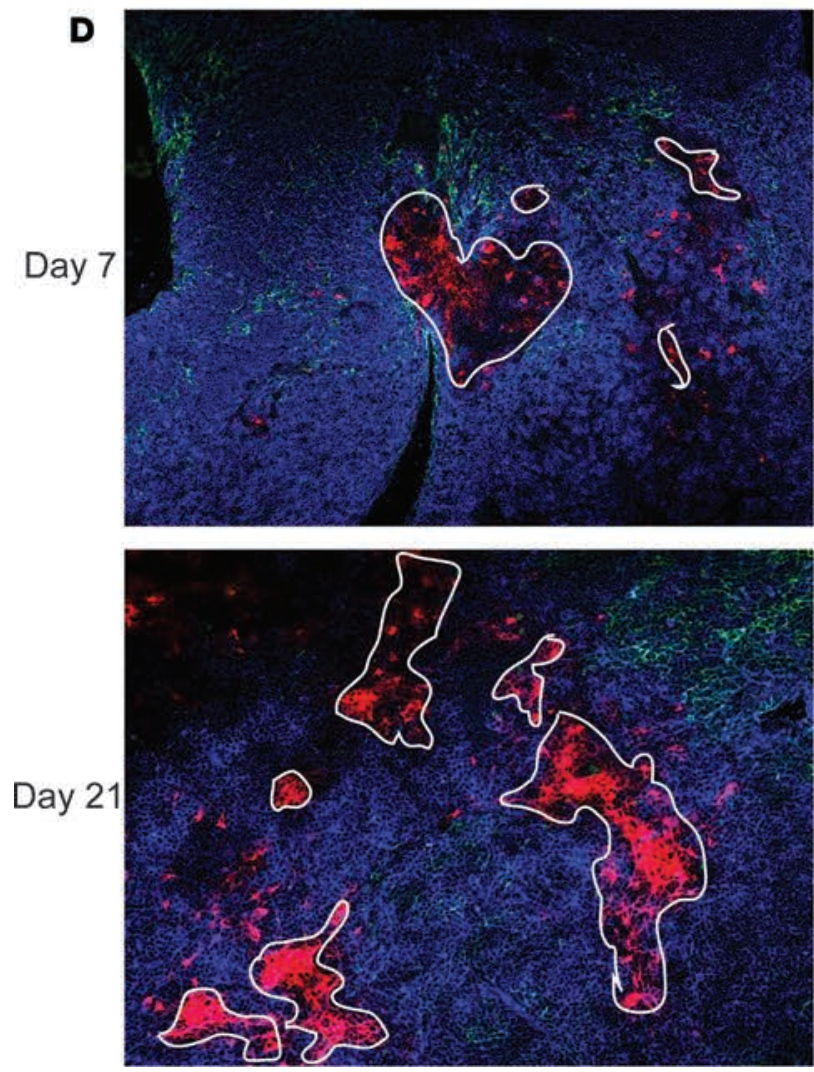

C

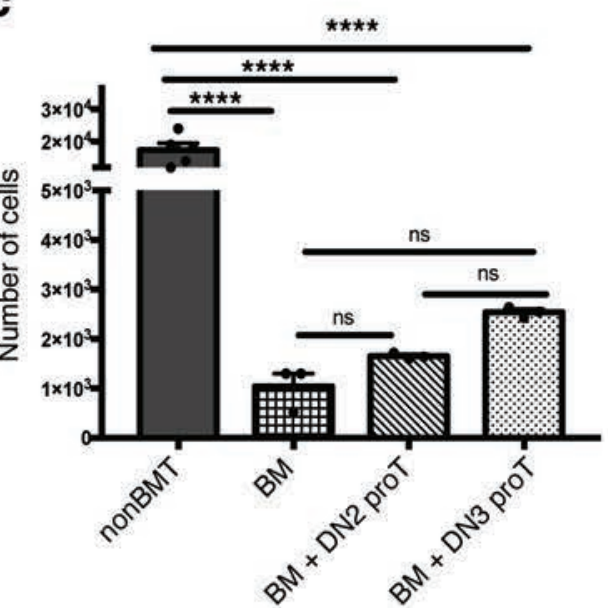

E
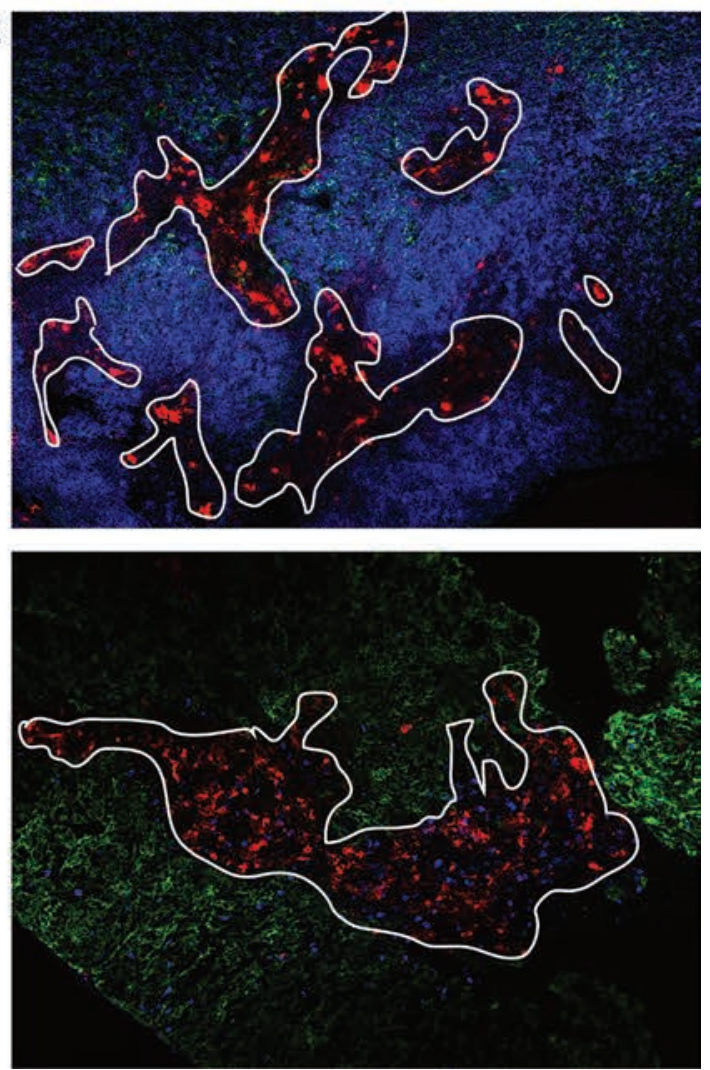

Figure 2. Double-negative 3 T cell progenitors stimulate mTEC recovery. Lethally irradiated CD45.1 recipients were given CD45.2 WT BM alone, with double-negative 2 (DN2) Thy1.1 progenitors (proTs) or with DN3 Thy1.1 proTs. Thymi were harvested at day 21, and their thymic epithelial cells (TECs) were analyzed by flow cytometry. Data are representative of 3 separate experiments, with 3-6 animals per group per time point. Data are shown as mean \pm SEM. Data sets were compared using 2-way ANOVA. ${ }^{*} P \leq 0.05$, ${ }^{* *} P \leq 0.001$, ${ }^{* * * *} P \leq 0.0001$. (A) Representative bulk proTs, DN2-sorted 
proTs, and DN3-sorted proTs. Live cells were selected, and flow cytometry plots show the DN populations of the bulk proTs (left) and DN2 proTs (middle), defined as (CD19, B220, Gr-1, CD11b, CD11c, Ter119, NK1.1)-CD45+CD4-CD8-CD25+CD44 hi . DN3 proTs (right) were gated on live cells and then defined as (CD19, B220, Gr-1, CD11b, CD11c, Ter119, NK1.1)-CD45+CD4-CD8-CD25+CD44 ${ }^{10}$. (B) Absolute numbers of mTECs at day 21 after transplant. (C) Absolute numbers of cTECs at day 21 after transplant. (D) DN2 proTs or (E) DN3 proTs (Thy1.1+, blue) in the cortex (BP-1, green) and in the medulla (UEA-1, red, outlined in white) at days 7 and 21 after transplant.

which could be cell intrinsic or due to radiation-induced damage to the LN microenvironment. Consistent with early thymopoiesis, DN2 cells were found at a statistically higher percentage in the cortex following irradiation $(P=0.0447$, Figure $3 \mathrm{E})$. Although not significant, there was a subsequent increasing trend of DN3 cells migrating into the medulla (Figure 3E). Interestingly, in vitro-derived proTs at the DN3 stage express significantly higher levels of CCR9 than those at the DN2 stage or thymocytes developing in vivo at either the DN2 or DN3 stage (Figure 3F and Supplemental Figure 2C). ProT-derived peripheral T cells expressed similar levels of CD3 as BM-derived and residual host T cells (Supplemental Figure 2, A and B). Taken together, these results indicate that DN3 is the optimum subset to increase peripheral $\mathrm{T}$ cells long term after transplant, possibly because of the ability of DN3 proTs to more rapidly boost mTEC numbers, while DN2 proTs provided a greater short-term increase in thymic size, as evidenced by thymocyte number on day 21 (Figure $3, \mathrm{~A}$ and $\mathrm{C}$ ).

$T E C$ recovery is limited in the absence of developing $T$ cells. We have shown that improvement in short-term thymopoiesis achieved by adoptively transferred proTs is correlated with an increase in the number of thymically located proTs as compared with BM-only controls (see Figure 3A), whereas increases in elements of the thymic microenvironment, namely mTECs, are correlated with the specific locality of those adoptively transferred proTs within the thymus (Figure 2, B, D, and E). However, in those studies, each group had contributions to thymic recovery from progenitors recruited from the BM graft. While the total number of cells in the thymus peaked at day 21 (Figure 1B), the number of DN progenitors within the thymus peaked at day 14 following adoptive transfer (Figure 4A). The increase in DN cells in the thymus is not only due to the proT-derived cells, but also to increased recruitment of lymphoid progenitors from the engrafted BM (40-42). In fact, progenitors recruited from the BM are known to actively compete with more mature progenitors in the thymus. When comparing mice given either WT or Rag-/- BM with or without proTs, mice that received WT BM with proTs had increased numbers of DN cells overall as compared with those that received WT BM alone, as did mice that received WT BM alone when compared with mice that received Rag-/- BM alone (Figure 4A). When broken down by subset (Figure 4, B-D), this held especially true in DN3 and DN4 subsets (Figure 4, C and D), although a small minority of Rag -/- cells can differentiate past the DN3 stage under some conditions (43). There is less disparity in the DN2 subset of cells present in the thymi of mice transplanted with either WT or Rag-/- BM with or without proTs, the DN2 subset being less proliferative and at a stage of differentiation before the Rag gene is required for successful TCR rearrangements (Figure 4, B and C, and Supplemental Figure 3, A-D). Despite having shown that proT localization in the thymus was correlated with increased TEC recovery (Figure 2) and that progenitors from the BM of a WT graft also increased the number of DN cells within the thymus as compared with mice that received Rag-/- BM with proTs (Figure 4), we wanted to isolate the effects of the proTs from the effects of progenitors recruited from the BM graft on the thymic microenvironment. So, we transplanted Rag-/- BM with or without proTs and assessed TEC recovery and peripheral $\mathrm{T}$ cell reconstitution. The group that received the proTs showed a significant increase in the total TEC number on day 21 after transplant as compared with the group that received Rag-/- BM alone (Figure 5A). Additionally, the number of cycling TECs was also significantly increased by day 21 in the group that received the proTs, as measured by BrdU incorporation (Figure 5B) and expression of Ki67 (data not shown). Consistent with our previous experiments, the total increase in TECs was due to an increase in the number of mTECs (Figure 5C), while the proT therapy did not stimulate significant recovery of CTECs (Figure 5D). These results were confirmed by the proportion of immunofluorescent signal due to UEA-1 (Figure 5H), indicating the medulla, in confocal microscopy images of healthy (nonirradiated) control thymi (Figure 5E), thymi of recipients of Rag-/- BM (Figure 5F), and thymi of recipients of Rag-/- BM plus proTs (Figure 5G). However, without the continued recruitment of progenitors from the BM, TECs cease to proliferate (Figure $5 \mathrm{~B}$ ) and their numbers begin to decline after day 21 (Figure 5A).

In the model pairing proTs with Rag-/- BM, we saw a peak in the number of thymocytes at day 21 (Figure $6 \mathrm{~A}$ ) but no significant increase in mature peripheral $\mathrm{T}$ cells at this point (Figure 6, B and C). Looking 
Thymus

A

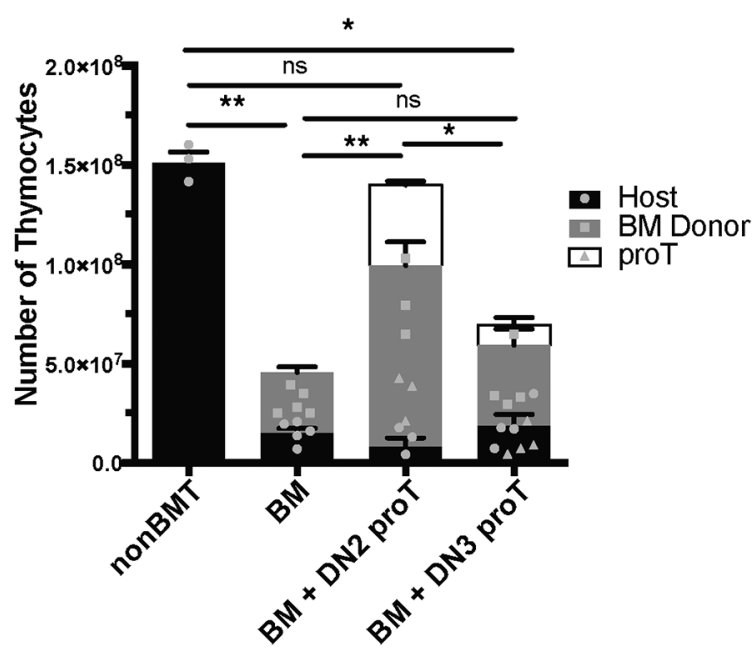

C

Day 60

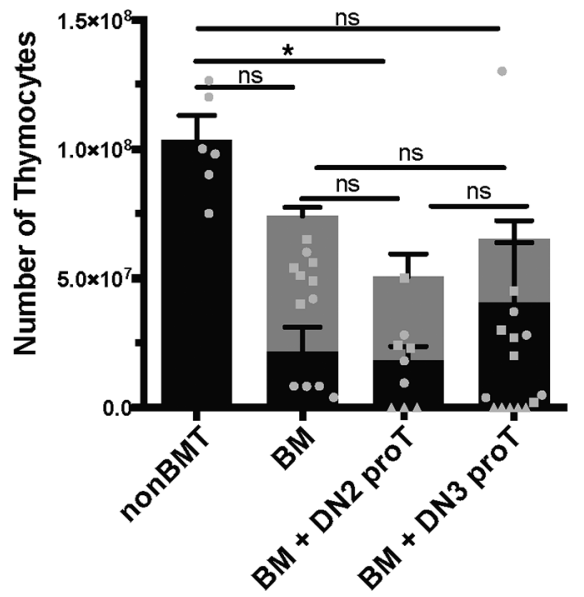

E

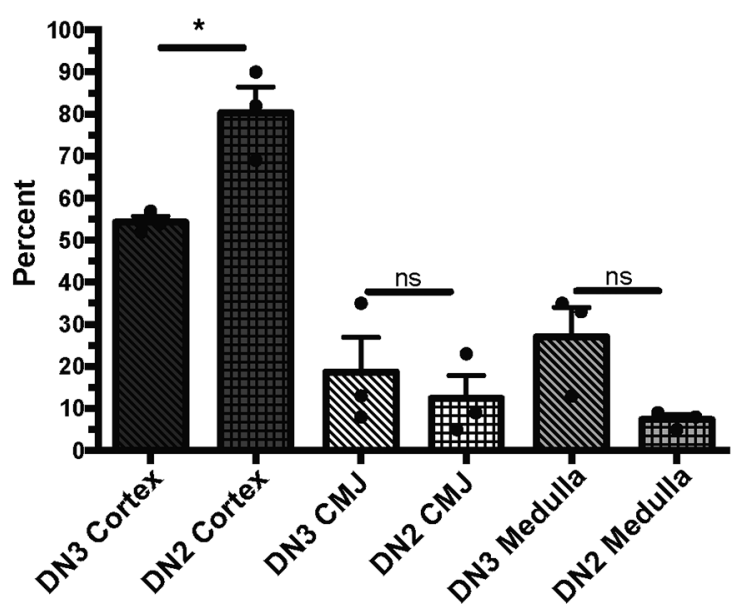

Spleen

B

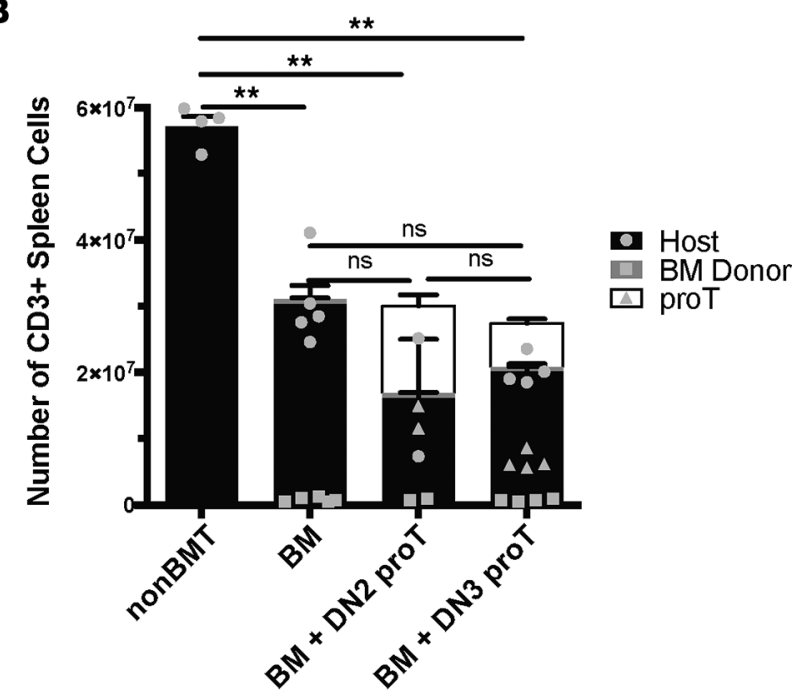

D

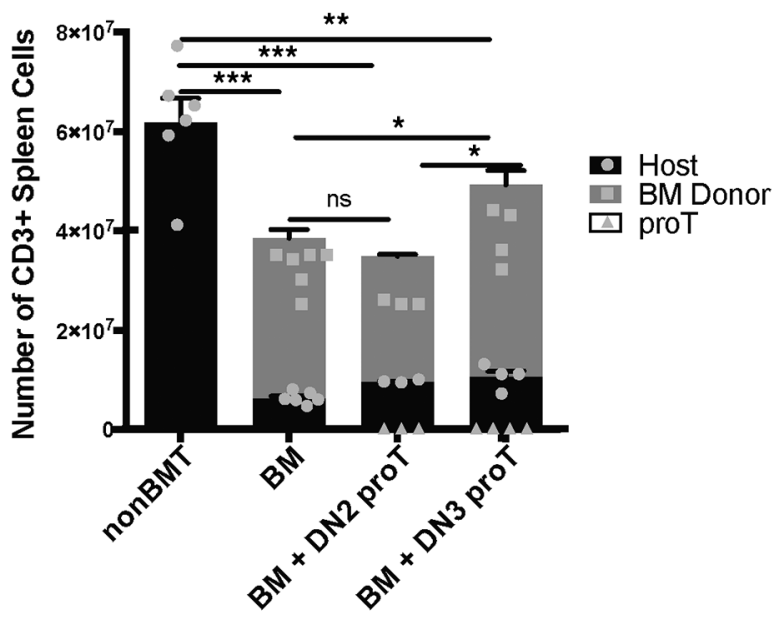

F

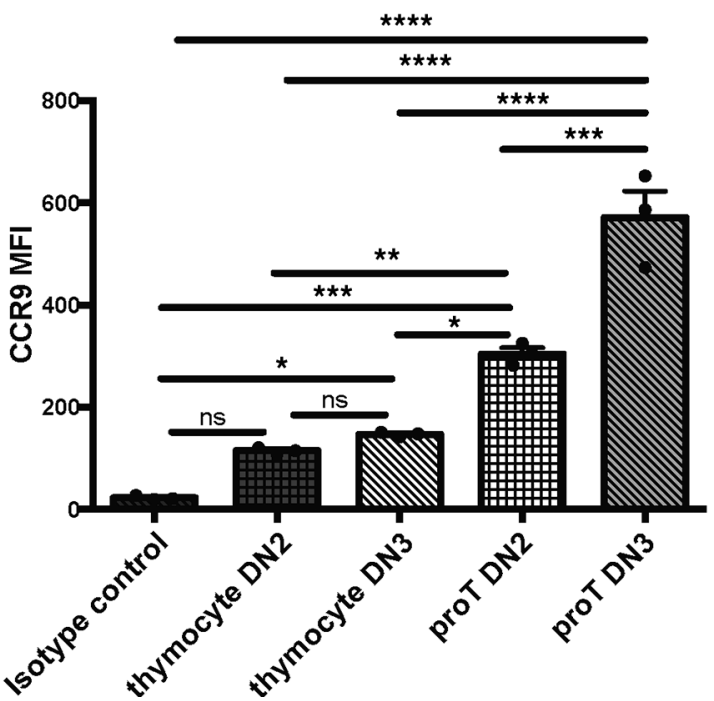

Figure 3. Double-negative $\mathbf{2} \mathrm{T}$ cell progenitors boost overall thymic size, and double-negative $\mathbf{3} \mathrm{T}$ cell progenitors lead to long-term $\mathrm{T}$ cell increases. Lethally irradiated CD45.1 B6 recipients were given CD45.2 WT BM alone, with double-negative 2 (DN2) Thy1.1 progenitors (proTs) or with DN3 Thy1.1 proTs. Thymi and peripheral lymphoid organs were harvested at day 21 and day 60 and were analyzed by flow cytometry. Data are representative of 3 separate experiments, with 4-6 mice per group per time point. Data are shown as mean $\pm \mathrm{SEM}$. Data sets were compared using 2 -way ANOVA. ${ }^{*} P \leq 0.05$, ${ }^{* *} P \leq$ $0.01,{ }^{* *} P \leq 0.001,{ }^{* * *} P \leq 0.0001$. (A) Absolute numbers of thymocytes at day 21 after transplant. (B) Absolute numbers of $\mathrm{CD}^{+}$spleen cells at day 21 
after transplant. (C) Absolute numbers of thymocytes at day 60 after transplant. (D) Absolute numbers of CD3+ spleen cells at day 60 after transplant. (E) Percentage of DN3 and DN2 cells present in the cortex, cortical medullary junction (CMJ), and medulla following irradiation and transfer of DN3 and DN2 cells onto thymic slices. (F) Median fluorescent intensity of CCR9 expressed on DN2 and DN3 in vivo thymocytes and in vitro proTs.

past day 21, proT-derived cells begin to exit the thymus (Figure 6D) and migrate to the periphery by day 35 , preferentially residing in the spleen (Figure 6, E and F). The CD4 and CD8 profiles at days 21, 35, and 60 are shown in Supplemental Figure 4. The egress to the periphery correlated with the loss of the beneficial effects of proTs on TECs (Figure 5, A and B). By day 60, we observed no continued benefit to the TEC (Figure 5A), thymocyte (Figure 6G), or peripheral T cell (Figure 6, H and I) compartments. Taken together, these data indicate that TECs damaged by radiation can be stimulated to proliferate by the presence of developing $\mathrm{T}$ cells within the thymus but that this effect is temporary in the absence of a continued influx of progenitors from the BM.

\section{Discussion}

The presence of thymic progenitors within the thymus is essential to the health of the microenvironment under normal physiological conditions and even more so when recovery from damage is required. In fact, thymic size is regulated by a feedback loop responding to the number of DN precursors and their competition for stromal niches (40). This concept is clearly illustrated by work from the van den Brink group, who first utilized the OP9-DL1 cell coculture system to produce proTs for the purpose of enhancing thymic recovery from radiation damage (32). An allogeneic BMT model was used to show that proTs enhanced thymic reconstitution and produced a broad repertoire of functional mature peripheral $\mathrm{T}$ cells, which protected against immunological challenges $(32,33)$. Furthermore, human in vitro-generated proTs have demonstrated the ability to enhance thymopoiesis after transplant in mice (44). These studies have set the foundation for future clinical trials using human proT protocols.

As these protocols are developed $(27,30,31)$, it is important to select the optimal cell product to expand and infuse. To obtain preclinical data that would direct clinical protocols, we used a congenic BMT model to isolate the effects of proT therapy on posttransplant reconstitution from any confounding factors due to allogeneic reactivity, which can impede positive and negative selection (45). We first examined the homing of in vitro-derived proTs to and within the thymus and their persistence in the periphery. By monitoring luciferase signal from proTs, we were able to track their homing to the thymus and egress to the periphery, establishing that numbers of proT-derived cells peaked thymically and in total at day 21 , following which there was a gradual decline, reaching a steady-state level in the periphery. Looking at the movement of these cells within the thymus, we found that proTs were not able to migrate normally on irradiated stromal cells as compared with healthy stromal cells, a trend that was also seen in WT thymocytes on the same stromal cells. These data indicate a disruption in the function of the thymic microenvironment, which agrees with the current literature indicating a defect in thymic progenitor homing as a result of disrupted chemokine production following radiation-induced damage $(34,46)$. This highlights a potential hurdle to the effectiveness of this therapy and also provides a window of time in which strategies to improve thymic homing may be most effective for optimizing proT therapy.

In the current study, we focused on DN3 cells in our homing and migration studies, in contrast to previous studies focusing on proT therapy in the DN2 subset (32). We sought to delineate the differential effects that these two subsets might have on the thymic microenvironment. We found that adoptive transfer of DN2 cells preferentially increased the lymphopoietic compartment of the thymus, while adoptive transfer of DN3 cells stimulated recovery of mTECs, which was associated with a long-term increase in the numbers of $\mathrm{T}$ cells found in the spleen. Consistent with this hypothesis was the localization of purified DN2 cells to the cortex in significantly higher numbers than DN3 cells following transfer to irradiated thymus slices and a subsequent increase of DN3 cell egress to the medulla. In this instance the increase was not statistically significant but an upward trend (Figure 3). The reason for these divergent outcomes may lie in the fact that the DN3 cells retained localization within the medulla of the thymus longer than the DN2 cells. Presumably this would facilitate more interactions between the proTs and mTECs. This crosstalk provides important stimulatory signals for mTECs, which turn over every 10 to 14 days (47, $48)$, through receptor activator of NF- $\mathrm{B}$, RANKL, CD80/86, LT $\beta R$, and CD40 (26, 49-52). The difference in effects on short-term and long-term thymopoiesis and TEC recovery seen between differing input populations of proTs would suggest that using a combination of DN2 and DN3 proTs may be an optimum approach. While mature proT-derived $\mathrm{T}$ cells are present in peripheral lymphoid organs soon after 

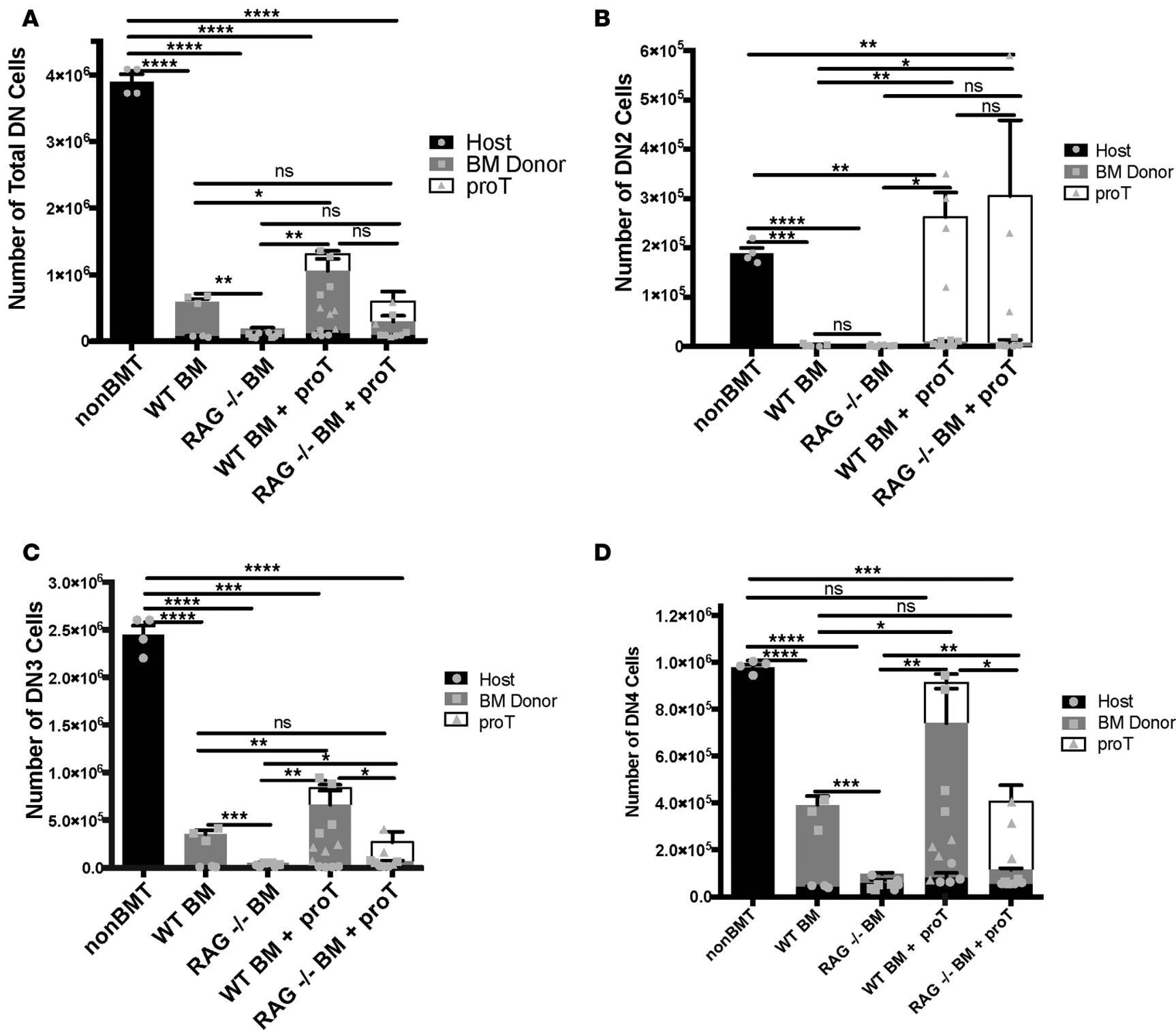

Figure 4. Numbers of double-negative cells are additively increased by T cell progenitor therapy. Lethally irradiated CD45.1 B6 recipients were given CD45.2 WT or Rag-/- BM with or without Thy1.1+ progenitors (proTs). Thymi were harvested at the day 14, and their double-negative (DN) T cell progenitors were analyzed by flow cytometry. Data are representative of 2 separate experiments, with $3-5$ animals per group. Data are shown as mean \pm SEM. Data sets were compared using 2-way ANOVA. ${ }^{*} P \leq 0.05,{ }^{* *} P \leq 0.01$, ${ }^{* *} P \leq 0.001$, ${ }^{* * * *} P \leq 0.0001$. (A) Total numbers of DN cells in the thymus. (B) Total numbers of DN2 cells in the thymus. (C) Total numbers of DN3 cells in the thymus. (D) Total numbers of DN4 cells in the thymus.

transplant but are likely progressively diluted out, BM-derived $\mathrm{T}$ cells in peripheral lymphoid organs are increased more long term. Presumably both the proTs and BM-derived peripheral mature T cells would enhance protection against infection, but the contributions of each cannot be effectively separately tested. Theoretically, the DN2 component of this approach would result in a short-term increase in thymic size, with a resultant early wave of protective peripheral $\mathrm{T}$ cells, while the DN3 subset would supplement long-term repair of the thymic microenvironment associated with a long-term boost in peripheral $\mathrm{T}$ cell numbers. As such, our work has implications for future clinical protocols.

Similarly, we observed a direct effect on the proliferative state of TECs in relation to the presence or absence of proTs within the thymus by using a model in which the BM graft (Rag-/-) was incapable of producing proTs that could successfully complete differentiation. When proTs were given Rag-/- BM, we observed a significant but transient increase in the proliferation and numbers of TECs, demonstrating the importance of continued strong recruitment of progenitors from the BM and the resultant crosstalk between immature T cells and TECs (24). While proT therapy does enhance recruitment on its 
A

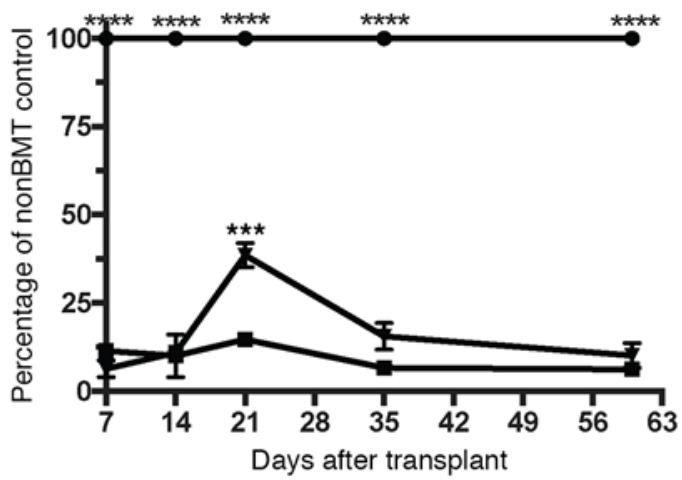

B

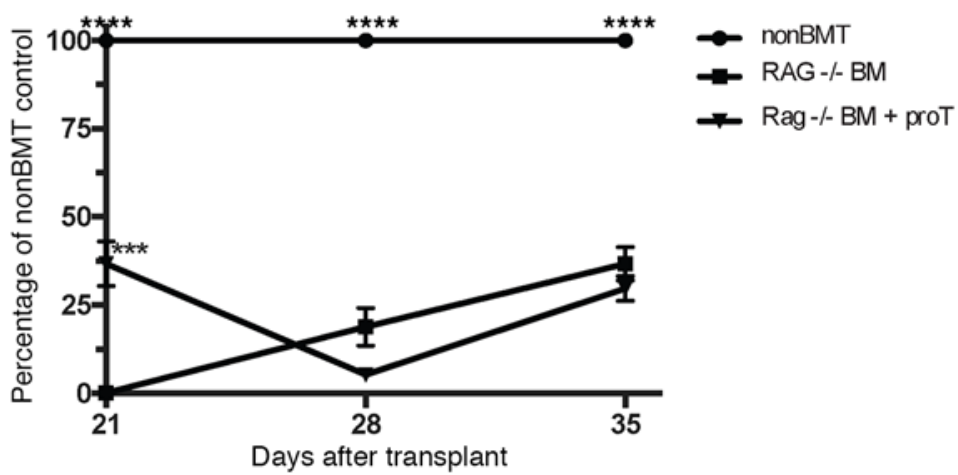

nonBMT

$R A G-/ B M$

RAG -/- BM+ proT
$\mathbf{F}$

E
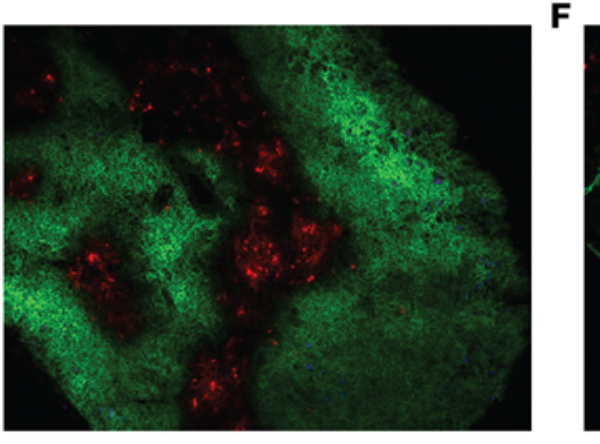

H

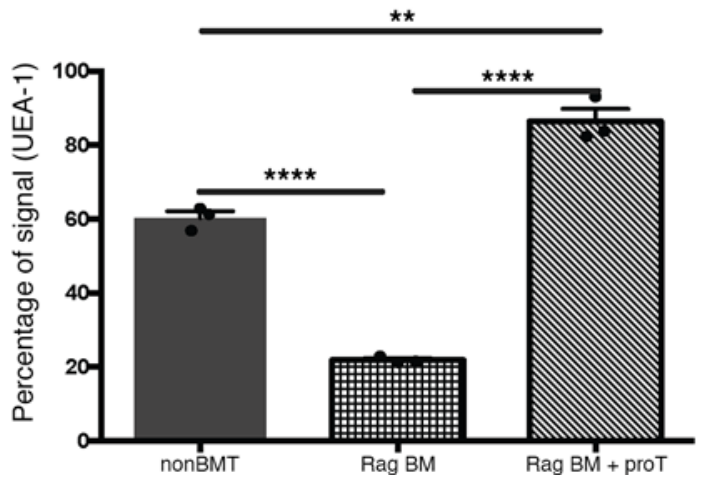

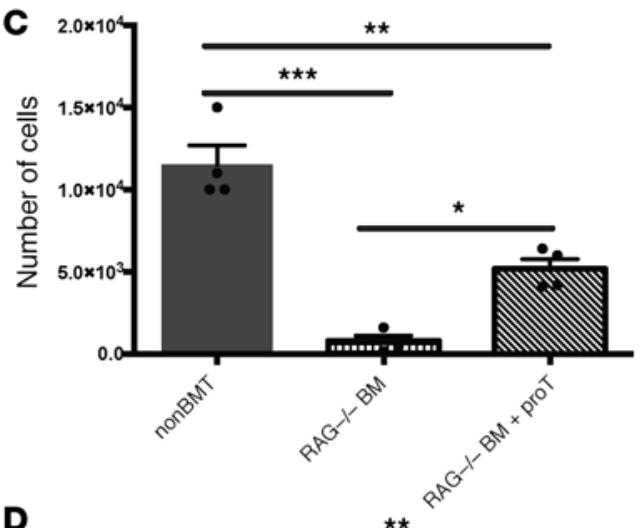

D

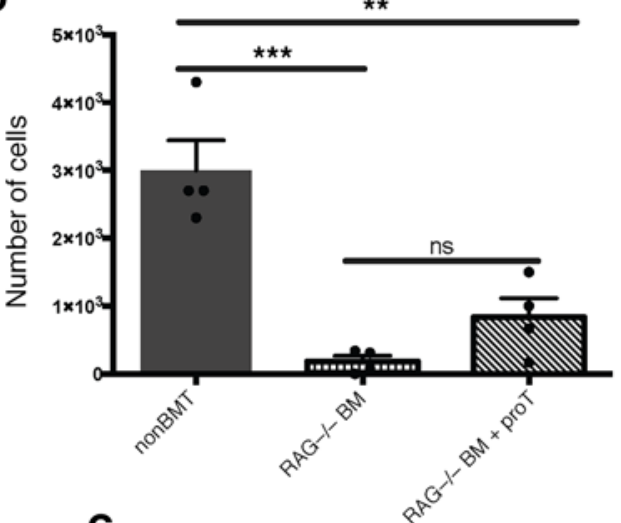

G

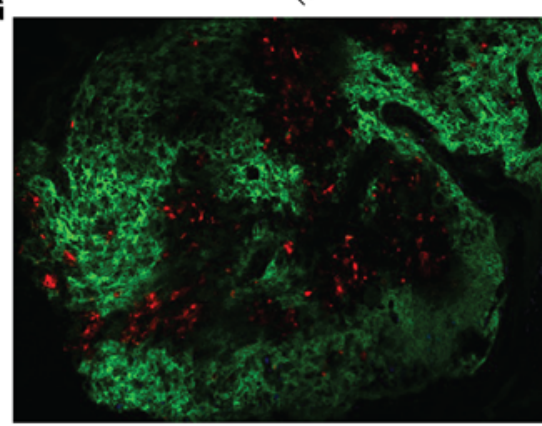

Figure 5. T cell progenitors without T cell-competent BM stimulate temporary medullary thymic epithelial cell recovery. Lethally irradiated $\mathrm{CD} 45.1 \mathrm{~B} 6$ recipients were given CD45.2 Rag-/- BM with or without Thy1.1 progenitors (proTs). Thymi were harvested at the indicated time points, and their thymic epithelial cells were analyzed by flow cytometry. Data are representative of 3 separate experiments, with 3-5 animals per group per time point. Data are shown as mean \pm SEM. Data sets were compared using 2-way ANOVA. ${ }^{*} P \leq 0.05,{ }^{* *} P \leq 0.01,{ }^{* *} P \leq 0.001$, ${ }^{* * *} P \leq 0.0001$. (A) Numbers of thymic epithelial cells expressed as a percentage of a non-BMT controls at the indicated time points. (B) Numbers of BrdU+ thymic epithelial cells expressed as a percentage of a non-BMT controls at the indicated time points. (C) Absolute numbers of medullary thymic epithelial cells at day 21 after transplant. (D) Absolute numbers of cortical thymic epithelial cells at day 21 after transplant. (E) UEA-1 (red) and BP-1 (green) staining of thymic sections at day 21 of nontransplanted controls. (F) Rag-/-BM recipients and (G) recipients of Rag-/- BM and proTs. Original magnification, $\times 10$. (H) Quantification of UEA-1 immunofluorescent signal.

own, it is reasonable to speculate that better recovery of the thymic microenvironment may be achieved by combining proT therapy with other strategies to enhance recruitment, such as pretreatment of donor marrow with thymic homing-associated chemokines (46). This may improve the insufficient support of thymic homing resulting from radiation damage to the microenvironment $(53,54)$. Therefore, by better understanding critical factors responsible for the effectiveness of proT therapy, we can more successfully adapt it to the clinic and achieve superior patient outcomes. 
A
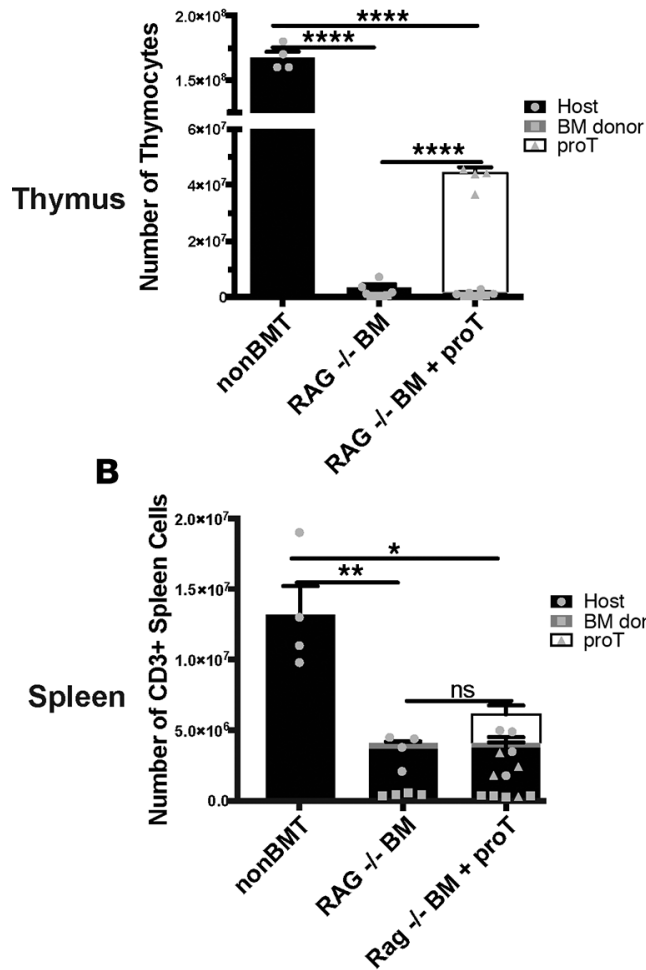

C

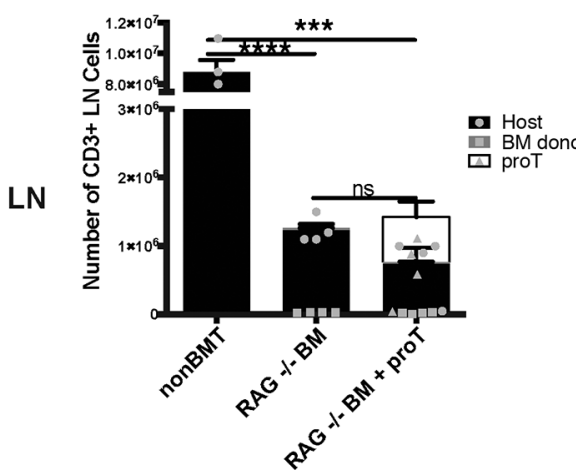

D

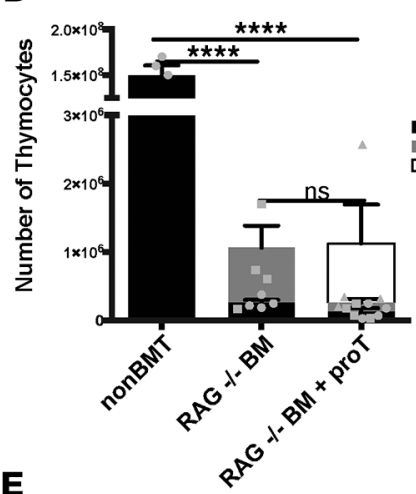

E

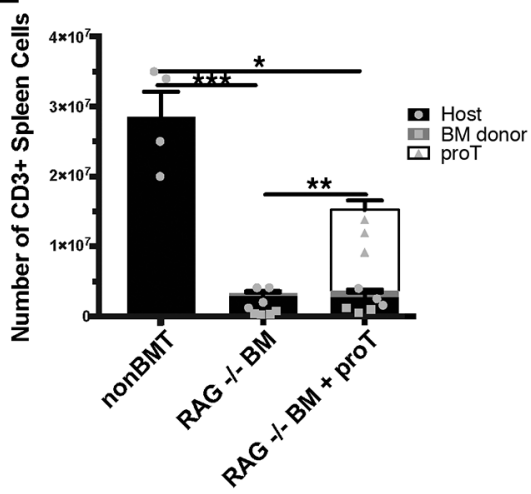

$\mathbf{F}$

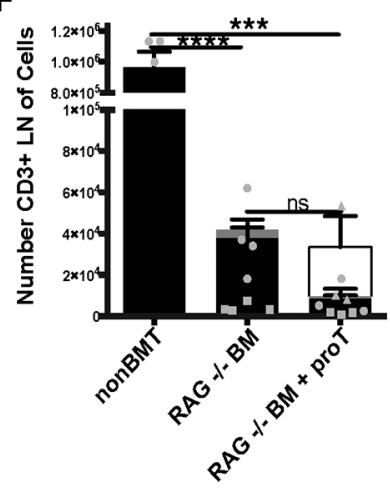

G
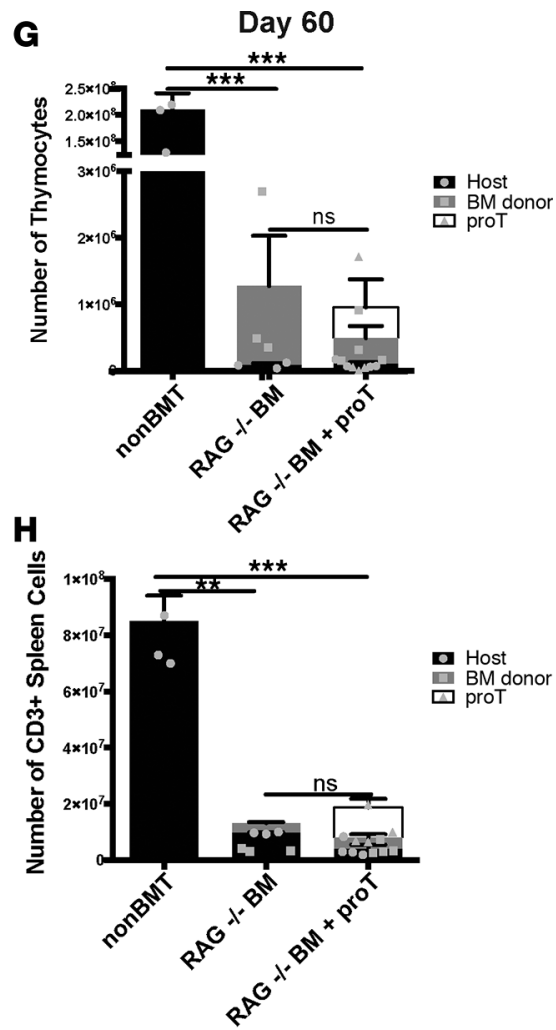

I

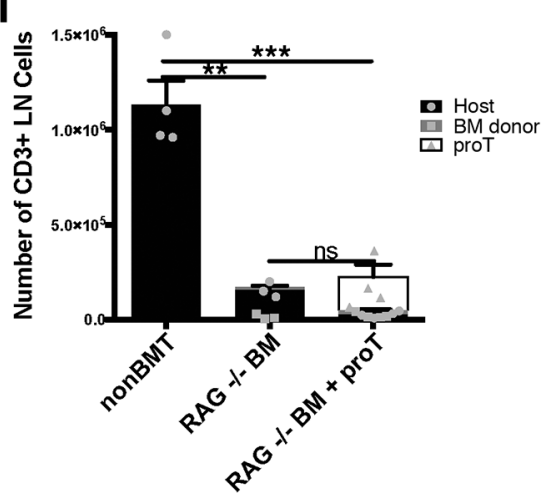

Figure 6. Without T cell-competent marrow, T cell gains from T cell progenitor therapy are temporary. Lethally irradiated CD45.1 B6 recipients were given CD45.2 Rag-/- BM with or without Thy1.1 progenitors (proTs). Thymi, spleens, and lymph nodes (LNs) were harvested at the indicated time points and were analyzed by flow cytometry. Data are representative of 3 separate experiments, with $3-5$ animals per group per time point. Data are shown as mean \pm SEM. Data sets were compared using 2 -way ANOVA. ${ }^{*} P \leq 0.05,{ }^{* *} P \leq 0.01,{ }^{* *} P \leq 0.001,{ }^{* * * *} P \leq 0.0001$. (A, D, and $\left.\mathbf{G}\right)$ Numbers of total thymocytes at the indicated time points after transplant. (B, E, and $\mathbf{H}$ ) Numbers of total CD $^{+}$spleen cells at the indicated time points after transplant. (C, $\mathbf{F}$, and I) Numbers of total $\mathrm{CD}^{+} \mathrm{LN}$ cells at the indicated time points after transplant.

\section{Methods}

Mice. Mice were used at 8 to 12 weeks of age and were all female. C57BL/6 (B6) mice were purchased from Charles River Laboratories. Congenic B6 CD45.1 mice were purchased from the National Cancer Institute. Congenic B6 Thy1.1 mice were purchased from Jackson Laboratories. B6 luciferase-transgenic mice, provided by Christopher Contag (Stanford University, Stanford, California, USA) (55), were bred in our animal colony under the guidance of in-house veterinary staff. Mice were housed in a specific pathogen-free facility.

Isolation of hematopoietic progenitor cells. BM was collected from femurs and tibiae of B6 Thy1.1 mice after crushing them with a mortar and pestle, followed by red blood cell lysis for 5 minutes at room temperature using PharmLyse (BD). Lineage-positive cells were depleted using a mouse lineage cell depletion kit (Miltenyi Biotec) and then enriched for Sca1 using the EasySep Mouse SCA1 Positive Selection Kit (Stem Cell Technologies). 
OP9-DL1 cultures. OP9-DL1 cells were provided by the Zúñiga-Pflücker lab. OP9-DL1 cultures and OP9DL1/hematopoietic progenitor cell cocultures were maintained as previously described (56). Briefly, hematopoietic progenitor cells were seeded onto 70\%-90\% confluent OP9-DL1 10-cm dishes at $1 \times 10^{6}$ per dish in the presence of $5 \mathrm{ng} / \mathrm{ml}$ Flt3L (R\&D systems) and $5 \mathrm{ng} / \mathrm{ml} \mathrm{IL-7} \mathrm{(Peprotech).} \mathrm{After} 7$ days, nonadherent cells were harvested and split 1:4 (from 1 dish onto 4 dishes containing new OP9-DL1). Phenotype was assessed by flow cytometry. Nonadherent cells were harvested on day 10 and used for transplant or 2-photon imaging.

HSCT congenic model with proT adoptive transfer. B6 recipients were given 11 Gy total body irradiation using an X-RAD 320 (Precision X-Ray) on day -1. On day 0, recipients were injected intravenously with 5 $\times 10^{6} \mathrm{~T}$ cell-depleted B6 CD45.1 BM or Rag-/- BM cells. T cell depletion was accomplished by incubating the marrow with antibodies against CD4 (GK1.5, National Cell Culture Center) and CD8 (2.43, National Cell Culture Center) at $0.02 \mathrm{mg} / \mathrm{ml}$ on ice for 30 minutes, followed by the addition of rabbit serum complement at $10 \% \mathrm{v} / \mathrm{v}$ and incubation at $37^{\circ} \mathrm{C}$ for 45 minutes. The cells were then washed and counted. Some groups also received $8 \times 10^{6}$ bulk proTs, DN2-sorted proTs $\left(\mathrm{CD}^{-} 5^{+} \mathrm{CD} 4^{-} \mathrm{CD} 8^{-} \mathrm{CD} 11 \mathrm{~b}^{-} \mathrm{CD} 11 \mathrm{c}^{-} \mathrm{B} 220^{-} \mathrm{CD} 19-\right.$ NK1.1- ${ }^{-}$rr1-Ter119-CD25+CD44 ${ }^{+}$), or DN3-sorted proTs $\left(\mathrm{CD} 45^{+} \mathrm{CD} 4^{-} \mathrm{CD} 8^{-} \mathrm{CD} 11 \mathrm{~b}^{-} \mathrm{CD} 11 \mathrm{c}^{-} \mathrm{B} 220^{-} \mathrm{CD} 19\right.$ NK1.1-Gr1-Ter119-CD25+CD44-) intravenously on day 0.

Flow cytometry and cell sorting. For BrdU incorporation experiments, mice received $1 \mu \mathrm{g}$ BrdU intraperitoneally 1 hour before harvest. For all harvests, thymi, spleens, and LNs were harvested at various time points after transplant, processed into single-cell suspensions, and analyzed by flow cytometry. TECs were isolated as previously described (57). Briefly, thymi were harvested, nicked, and agitated to release hematopoietic cells. They were then transferred to media containing collagenase D and DNase, dissociated using the Program B setting on a Miltenyi GentleMACS tissue dissociator, and incubated for 15 minutes at $37^{\circ} \mathrm{C}$. This was repeated once with fresh media and then again with media containing collagenase/dispase and DNase. All samples were stained with a fixable viability dye conjugated to either ef780 or ef450 (eBioscience). Fixation and intracellular/intranuclear staining were performed using the eBioscience Foxp3 staining kit. The following antibodies were purchased from BD Biosciences: CD45 (30-F11), CD3 (500A2), CD8 (53-6.7), cKit (2B8), CD62L (MEL-14), CD11b (M1/70), CD11c (HL3), Ter119 (TER119), CD19 (1D3), B220 (RA3-6B2), NK1.1 (PK136), Gr-1 (RB6-8C5), MHCII (M5/114), EpCAM (G8.8), CD45.1 (A20), Thy1.1 (OX-7), and BrdU (3D4). The following antibodies were purchased from eBioscience: CD45.2 (clone 104), CD44 (IM7), CD4 (GK1.5), CD25 (7D4), TCR $\beta$ (H57-597), TCR $\gamma \delta$ (GL-3), BP-1 (6C3), and Ki67 (SolA15). UEA-1 (B-1065) was purchased from Vector Laboratories. Single-cell suspensions were interrogated using a BD LSRFortessa flow cytometer. Data were analyzed using FlowJo V10 (Treestar Inc.).

In vivo bioluminescent imaging. In vivo bioluminescent imaging was performed as described previously (58). Mice were injected with firefly luciferin substrate $(0.1 \mathrm{ml} ; 30 \mathrm{mg} / \mathrm{ml}$; Promega) intraperitoneally. After 5 minutes, a Xenogen IVIS imaging system was then used to measure luminescent quantification under "auto" exposure. Data were analyzed and quantified with Living Image 3.0 software (Calipers).

Confocal microscopy. Thymi were immersed in OCT Compound for Cryostat Sectioning (Tissue-Tek) in plastic molds and frozen in liquid nitrogen. Thymi were cut into 6-micron sections on a cryostat, fixed in room temperature acetone for 5 minutes, and rehydrated with $1 \times$ PBS. Sections were blocked with the Avidin/Biotin blocking kit (Vector Laboratories) according to the kit's protocol and then blocked with 10\% normal donkey serum (Jackson Immunoresearch). Primary antibodies used were CD90.1 AF647 (Thy1.1) (Biolegend), BP-1 FITC (eBioscience), and UEA-1 Biotinylated (Vector Laboratories). Secondary antibody Streptavidin Cy3 (Jackson Immunoresearch) was used. Slides were coverslipped with hard set DAPI (Vector Labs) and examined by confocal fluorescence microscopy (Olympus FluoView FV1000 BX61 Upright Confocal, Olympus Optical). Images were analyzed using the color segmentation plugin in Fiji image processing software (ImageJ, NIH)

Two-photon microscopy. Sample preparation and 2-photon microscopy were performed as previously described (59). Cells labeled with either cell tracker violet or cell tracker orange (Thermo Fisher Scientific) were added to the slices and incubated overnight in complete RPMI at $5 \% \mathrm{CO}_{2}$ and $37^{\circ} \mathrm{C}$. Samples were excited with a MaiTai TiSaphire DeepSee HP laser (15 W; Spectra-Physics) at $870 \mathrm{~nm}$, and emissions of 440-480 nm (CTV), 500-520 nm (GFP), and 560-630 nm (CTO) were collected. A 4-channel Leica TCS MP microscope with a resonant scanner containing 2 NDD- and 2 HyD- photomultiplier tubes operating at video rate was used for image capture. Images acquired were 50-100 $\mu \mathrm{m}$ below the top of the slice, and $512 \times 512 x-y$ frames were collected at $3.0-\mu \mathrm{m}$ steps every 30 seconds for 20 minutes. Image stacks were spectrally unmixed with the LAS AF 3.0 dye separation software module 
(Leica Microsystems). Three-dimensional images, time-lapse movies, and cell tracks were generated with Imaris $8.2 \times 64$ software (Bitplane), and tracks were verified manually. Tracking data were analyzed in Microsoft Excel with a custom macro as previously described (60).

Epifluorescent microscopy. Thymus lobes from irradiated mice were embedded in low-melt agarose and sliced into $800-\mu \mathrm{m}$ sections using a vibratome as previously described $(59,61)$. CD 45.1 DN2 and Thy $1.1 \mathrm{DN} 3$ thymocytes were sorted and incubated with the slices for 36 hours. The slices were then fixed with $2 \%$ PFA, dehydrated with $30 \%$ sucrose and then embedded/frozen in OCT. Cryosections of the slices were stained with Alexa Fluor 647 BP-1 (Biolegend), biotinylated UEA-1 (Vector Laboratories), PE CD45.1 (BD Biosciences), and BV480 Thy1.1 (BD Biosciences). Secondary streptavidin BV421 (Biolegend) was used. Images of the sections were tiled using a Leica DM6000B epifluorescent microscope. Images were spectrally unmixed with the LAS AF 3.0 dye separation software module (Leica Microsystems) and analyzed with Imaris $8.2 \times 64$ software (Bitplane). Surfaces for CD45.1 (DN2), Thy1.1 (DN3), and BP-1 (cortex) were generated, and distances to the cortices of DN2 and DN3 surfaces were calculated by the Imaris distance transformation function. Cells residing in the cortex were determined to be $0-1 \mu \mathrm{m}$ from the cortex surface, cells in the cortical medullary junction were determined to be $1-10 \mu \mathrm{m}$ from the cortex, and cells in the medulla were determined to be $>10 \mu \mathrm{m}$ from the cortex. Medulla-residing cells also had to display an UEA-1 MFI higher than background.

Statistics. Data are shown as mean \pm SEM. Data sets were compared using 2-way ANOVA. Significance was defined as $P<0.05$.

Study approval. Mice were used with the approval of the University of Minnesota institutional animal care and use committee.

\section{Author contributions}

MJS designed and performed experiments, analyzed data, and wrote the manuscript. DKR, SLP, and MJR performed experiments. JM and KCO performed experiments and analyzed data. HES, BTF, AB, KAH, $\mathrm{GAH}$, and JT provided guidance in experimental design and edited the manuscript. MM and JCZP provided guidance in experimental design, provided reagents, and edited the manuscript. BRB designed experiments and edited the manuscript.

\section{Acknowledgments}

We would like to acknowledge Patricia Taylor for her guidance and assistance in all mouse work. We would also to thank Leslie Jonart and Cordelia Dunai for their help with experiments and support. This work was supported in part by NIH grants P01 CA065493, R01 AI106791, and R01 AI081918 and Canadian Institutes of Health Research grant (MOP-119538). JCZP is supported by a Canada Research Chair in Developmental Immunology and by the Krembil Foundation.

Address correspondence to: Bruce R. Blazar, Pediatrics BMT, MMC 366 Mayo, 8366A (Campus Delivery Code), 420 Delaware Street SE, Minneapolis, Minnesota 55455, USA. Phone: 612.626.2734; E-mail: blaza001@umn.edu.

1. Pavletic SZ, et al. Unrelated donor marrow transplantation for B-cell chronic lymphocytic leukemia after using myeloablative conditioning: results from the Center for International Blood and Marrow Transplant research. J Clin Oncol. 2005;23(24):5788-5794.

2. Bladé J, et al. Criteria for evaluating disease response and progression in patients with multiple myeloma treated by high-dose therapy and haemopoietic stem cell transplantation. Myeloma Subcommittee of the EBMT. European Group for Blood and Marrow Transplant. Br J Haematol. 1998;102(5):1115-1123.

3. Locasciulli A, et al. Outcome of patients with acquired aplastic anemia given first line bone marrow transplantation or immunosuppressive treatment in the last decade: a report from the European Group for Blood and Marrow Transplantation (EBMT). Haematologica. 2007;92(1):11-18.

4. Wagner JE, et al. Bone marrow transplantation for recessive dystrophic epidermolysis bullosa. NEngl J Med. 2010;363(7):629-639.

5. Weinberg K, et al. Factors affecting thymic function after allogeneic hematopoietic stem cell transplantation. Blood. 2001;97(5):1458-1466.

6. Welniak LA, Blazar BR, Murphy WJ. Immunobiology of allogeneic hematopoietic stem cell transplantation. Annu Rev Immunol. 2007;25:139-170

7. Douek DC, et al. Assessment of thymic output in adults after haematopoietic stem-cell transplantation and prediction of T-cell reconstitution. Lancet. 2000;355(9218):1875-1881.

8. Hakim FT, et al. Age-dependent incidence, time course, and consequences of thymic renewal in adults. J Clin Invest. 
2005;115(4):930-939.

9. Williams KM, Hakim FT, Gress RE. T cell immune reconstitution following lymphodepletion. Semin Immunol. 2007;19(5):318-330

10. Wils EJ, et al. Insufficient recovery of thymopoiesis predicts for opportunistic infections in allogeneic hematopoietic stem cell transplant recipients. Haematologica. 2011;96(12):1846-1854.

11. Velardi E, Dudakov JA, van den Brink MR. Clinical strategies to enhance thymic recovery after allogeneic hematopoietic stem cell transplantation. Immunol Lett. 2013;155(1-2):31-35.

12. Waggoner JJ, Soda EA, Deresinski S. Rare and emerging viral infections in transplant recipients. Clin Infect Dis. 2013;57(8):1182-1188

13. Adkins B, Gandour D, Strober S, Weissman I. Total lymphoid irradiation leads to transient depletion of the mouse thymic medulla and persistent abnormalities among medullary stromal cells. J Immunol. 1988;140(10):3373-3379.

14. Chung B, Barbara-Burnham L, Barsky L, Weinberg K. Radiosensitivity of thymic interleukin-7 production and thymopoiesis after bone marrow transplantation. Blood. 2001;98(5):1601-1606.

15. Su DM, Navarre S, Oh WJ, Condie BG, Manley NR. A domain of Foxn1 required for crosstalk-dependent thymic epithelial cell differentiation. Nat Immunol. 2003;4(11):1128-1135.

16. Williams KM, et al. CCL25 increases thymopoiesis after androgen withdrawal. Blood. 2008;112(8):3255-3263.

17. Gossens K, et al. Thymic progenitor homing and lymphocyte homeostasis are linked via S1P-controlled expression of thymic P-selectin/CCL25. J Exp Med. 2009;206(4):761-778.

18. Petrie HT, Zúñiga-Pflücker JC. Zoned out: functional mapping of stromal signaling microenvironments in the thymus. Annu Rev Immunol. 2007;25:649-679.

19. Alves NL, et al. Characterization of the thymic IL-7 niche in vivo. Proc Natl Acad Sci USA. 2009;106(5):1512-1517.

20. Starr TK, Jameson SC, Hogquist KA. Positive and negative selection of T cells. Annu Rev Immunol. 2003;21:139-176.

21. Min D, et al. Protection from thymic epithelial cell injury by keratinocyte growth factor: a new approach to improve thymic and peripheral T-cell reconstitution after bone marrow transplantation. Blood. 2002;99(12):4592-4600.

22. Kelly RM, et al. Keratinocyte growth factor and androgen blockade work in concert to protect against conditioning regimen-induced thymic epithelial damage and enhance T-cell reconstitution after murine bone marrow transplantation. Blood. 2008;111(12):5734-5744.

23. Kelly RM, et al. Short-term inhibition of p53 combined with keratinocyte growth factor improves thymic epithelial cell recovery and enhances T-cell reconstitution after murine bone marrow transplantation. Blood. 2010;115(5):1088-1097.

24. Holländer GA, et al. Developmental control point in induction of thymic cortex regulated by a subpopulation of prothymocytes. Nature. 1995;373(6512):350-353.

25. Irla M, et al. Autoantigen-specific interactions with CD4+ thymocytes control mature medullary thymic epithelial cell cellularity. Immunity. 2008;29(3):451-463.

26. Desanti GE, et al. Developmentally regulated availability of RANKL and CD40 ligand reveals distinct mechanisms of fetal and adult cross-talk in the thymus medulla. J Immunol. 2012;189(12):5519-5526.

27. Schmitt TM, Zúñiga-Pflücker JC. Induction of T cell development from hematopoietic progenitor cells by delta-like-1 in vitro. Immunity. 2002;17(6):749-756

28. Schmitt TM, de Pooter RF, Gronski MA, Cho SK, Ohashi PS, Zúñiga-Pflücker JC. Induction of T cell development and establishment of T cell competence from embryonic stem cells differentiated in vitro. Nat Immunol. 2004;5(4):410-417.

29. de Pooter RF, Schmitt TM, Zúñiga-Pflücker JC. In vitro generation of T lymphocytes from embryonic stem cells. Methods Mol Biol. 2006;330:113-121.

30. Meek B, et al. In vitro-differentiated T/natural killer-cell progenitors derived from human CD34+ cells mature in the thymus. Blood. 2010;115(2):261-264.

31. Reimann C, et al. Human T-lymphoid progenitors generated in a feeder-cell-free Delta-like-4 culture system promote T-cell reconstitution in NOD/SCID/ $\gamma \mathrm{c}(-/-)$ mice. Stem Cells. 2012;30(8):1771-1780.

32. Zakrzewski JL, et al. Adoptive transfer of T-cell precursors enhances T-cell reconstitution after allogeneic hematopoietic stem cell transplantation. Nat Med. 2006;12(9):1039-1047.

33. Zakrzewski JL, et al. Tumor immunotherapy across MHC barriers using allogeneic T-cell precursors. Nat Biotechnol. 2008;26(4):453-461

34. Zlotoff DA, et al. Delivery of progenitors to the thymus limits T-lineage reconstitution after bone marrow transplantation. Blood. 2011;118(7):1962-1970.

35. Bhandoola A, von Boehmer H, Petrie HT, Zúñiga-Pflücker JC. Commitment and developmental potential of extrathymic and intrathymic T cell precursors: plenty to choose from. Immunity. 2007;26(6):678-689.

36. Brabb T, et al. Thymic stromal organization is regulated by the specificity of $\mathrm{T}$ cell receptor/major histocompatibility complex interactions. Eur J Immunol. 1997;27(1):136-146.

37. Manley NR. Thymus organogenesis and molecular mechanisms of thymic epithelial cell differentiation. Semin Immunol. 2000;12(5):421-428.

38. Germeraad WT, et al. Development of thymic microenvironments in vitro is oxygen-dependent and requires permanent presence of T-cell progenitors. J Histochem Cytochem. 2003;51(9):1225-1235.

39. Akiyama T, et al. The tumor necrosis factor family receptors RANK and CD40 cooperatively establish the thymic medullary microenvironment and self-tolerance. Immunity. 2008;29(3):423-437.

40. Prockop SE, Petrie HT. Regulation of thymus size by competition for stromal niches among early T cell progenitors. J Immunol. 2004;173(3):1604-1611

41. Martins VC, et al. Thymus-autonomous T cell development in the absence of progenitor import. J Exp Med. 2012;209(8):1409-1417.

42. Martins VC, et al. Cell competition is a tumour suppressor mechanism in the thymus. Nature. 2014;509(7501):465-470.

43. Petrie HT, Tourigny M, Burtrum DB, Livak F. Precursor thymocyte proliferation and differentiation are controlled by signals unrelated to the pre-TCR. J Immunol. 2000;165(6):3094-3098.

44. Awong G, et al. Human proT-cells generated in vitro facilitate hematopoietic stem cell-derived T-lymphopoiesis in vivo and restore thymic architecture. Blood. 2013;122(26):4210-4219. 
45. Krenger W, Blazar BR, Holländer GA. Thymic T-cell development in allogeneic stem cell transplantation. Blood. 2011;117(25):6768-6776.

46. Zhang SL, et al. Chemokine treatment rescues profound T-lineage progenitor homing defect after bone marrow transplant conditioning in mice. Blood. 2014;124(2):296-304.

47. Gray DH, et al. Developmental kinetics, turnover, and stimulatory capacity of thymic epithelial cells. Blood. 2006;108(12):3777-3785.

48. Gray D, Abramson J, Benoist C, Mathis D. Proliferative arrest and rapid turnover of thymic epithelial cells expressing Aire. $J$ Exp Med. 2007;204(11):2521-2528.

49. Williams JA, et al. Thymic medullary epithelium and thymocyte self-tolerance require cooperation between CD28-CD80/86 and CD40-CD40L costimulatory pathways. J Immunol. 2014;192(2):630-640.

50. Jenkinson SR, et al. TRAF3 enforces the requirement for T cell cross-talk in thymic medullary epithelial development. Proc Nat Acad Sci USA. 2013;110(52):21107-21112.

51. Lkhagvasuren E, Sakata M, Ohigashi I, Takahama Y. Lymphotoxin $\beta$ receptor regulates the development of CCL21-expressing subset of postnatal medullary thymic epithelial cells. J Immunol. 2013;190(10):5110-5117.

52. Hikosaka Y, et al. The cytokine RANKL produced by positively selected thymocytes fosters medullary thymic epithelial cells that express autoimmune regulator. Immunity. 2008;29(3):438-450.

53. Schwarz BA, Sambandam A, Maillard I, Harman BC, Love PE, Bhandoola A. Selective thymus settling regulated by cytokine and chemokine receptors. J Immunol. 2007;178(4):2008-2017.

54. Sanos SL, Nowak J, Fallet M, Bajenoff M. Stromal cell networks regulate thymocyte migration and dendritic cell behavior in the thymus. J Immunol. 2011;186(5):2835-2841.

55. Cao YA, et al. Shifting foci of hematopoiesis during reconstitution from single stem cells. Proc Natl Acad Sci USA. 2004;101(1):221-226.

56. Holmes R, Zúñiga-Pflücker JC. The OP9-DL1 system: generation of T-lymphocytes from embryonic or hematopoietic stem cells in vitro. Cold Spring Harb Protoc. 2009;2009(2):pdb.prot5156.

57. McLelland BT, Gravano D, Castillo J, Montoy S, Manilay JO. Enhanced isolation of adult thymic epithelial cell subsets for multiparameter flow cytometry and gene expression analysis. J Immunol Methods. 2011;367(1-2):85-94.

58. Aoyama K, et al. Inhibiting retinoic acid signaling ameliorates graft-versus-host disease by modifying T-cell differentiation and intestinal migration. Blood. 2013;122(12):2125-2134.

59. Dzhagalov IL, Melichar HJ, Ross JO, Herzmark P, Robey EA. Two-photon imaging of the immune system. Curr Protoc Cytom. 2012; Chapter 12:Unit12.26.

60. Zinselmeyer BH, et al. PD-1 promotes immune exhaustion by inducing antiviral T cell motility paralysis. J Exp Med. 2013;210(4):757-774.

61. Melichar HJ, Ross JO, Herzmark P, Hogquist KA, Robey EA. Distinct temporal patterns of T cell receptor signaling during positive versus negative selection in situ. Sci Signal. 2013;6(297):ra92. 